\title{
Severe iron deficiency anemia and anasarca edema due to excessive cow's milk intake
}

\author{
Elpis Mantadakis ${ }^{1}$, Panagiota Zikidou${ }^{1}$, Emmanouela Tsouvala ${ }^{2}$, Stavros Thomaidis ${ }^{1}$, \\ Athanassios Chatzimichael ${ }^{1}$ \\ ${ }^{1}$ Department of Pediatrics, Democritus University of Thrace Faculty of Medicine, ${ }^{2}$ Department of Neonatology, University \\ Hospital of Alexandroupolis, Thrace, Greece. \\ E-mail: emantada@med.duth.gr \\ Received:23rd November 2017, Revised: 3rd January 2018, Accepted: 25th February 2018
}

SUMMARY: Mantadakis E, Zikidou P, Tsouvala E, Thomaidis S, Chatzimichael A. Severe iron deficiency anemia and anasarca edema due to excessive cow's milk intake. Turk J Pediatr 2019; 61: 102-106.

The authors describe a 13-month-old girl who presented with progressively worsening anasarca edema that developed over the last three weeks along with increasing fatigue. Over the last several months she was consuming progressively increasing amounts of fresh cow's milk. Laboratory examinations on admission showed severe microcytic and hypochromic anemia (hemoglobin $3.8 \mathrm{~g} / \mathrm{dl}$ ) and hypoferritenemia indicative of iron deficiency, while urinalyses showed no proteinuria. The child was transfused with $13 \mathrm{ml} / \mathrm{kg}$ packed red blood cells and approximately $2 \mathrm{~g} / \mathrm{kg}$ intravenous albumin. On the second and fourth hospital days, she received $100 \mathrm{mg}$ of iron sucrose intravenously that she tolerated well. Eight months after the described events, she is healthy with normal hemoglobin for age, while she has no laboratory evidence of cow's milk protein allergy. Pediatricians should be aware of the association of severe iron deficiency anemia (IDA) and anasarca edema, and should screen infants in their practice for anemia at the age of 12 months or sooner, if risk factors are present.

Key words: anasarca edema, anemia, cow's milk, intravenous iron, iron deficiency.

Iron deficiency remains the most common nutritional disorder worldwide, despite its substantial decline in recent years because of the availability of iron-fortified infant formulas, of the use of iron supplements in exclusively breastfed infants beyond the $4^{\text {th }}$ month of life, and of the rising living standards. ${ }^{1,2}$ Based on a nationally representative cross-sectional health examination survey of the US population during the period 1988-1994, 9\% of toddlers aged 1 to 2 years and $9 \%$ to $11 \%$ of adolescent girls were iron deficient. ${ }^{3}$ Among them, iron deficiency anemia (IDA) is found in $3 \%$ and $2 \%$ to $5 \%$, respectively. ${ }^{3}$ More recently, the prevalence of iron deficiency among toddlers aged 1 to 2 years in the USA has been estimated at $13.5 \%$, and of IDA at $2.7 \% .4$

In developing countries, the prevalence of IDA is estimated to be much higher, although few data are available because accurate assessment of iron status is difficult, as it requires measurement of various hematologic and biochemical indicators that do not provide sufficient information alone and must be used in combination. Despite that, it is assumed that approximately $50 \%$ of the cases of anemia in developing countries are due to iron deficiency. ${ }^{5}$ The World Health Organization (WHO) considers countries with prevalence of anemia $\geq 40 \%$ to have a serious public health problem, and these countries are located in Africa, SouthEast Asia and Eastern Mediterranean with corresponding prevalence of anemia $67.6 \%$, $65.5 \%$, and $46.7 \%$, respectively, in the most recent WHO study of the period 1993-2005. ${ }^{5}$

One of the main risk factors for IDA in industrialized countries is the consumption of large amounts of unfortified cow's milk that is also frequently associated with occult gastrointestinal bleeding. Protein-losing 
enteropathy (PLE) is a clinical entity rarely associated with severe IDA. Because of the substantial decline in the incidence of IDA in recent decades, the link of PLE with IDA may not be immediately appreciated, and pediatricians are unlikely to be familiar with this association.

Therefore, we present a 13-month-old Caucasian female, who presented with anasarca edema due to severe IDA, describe her successful therapy with intravenous iron, and review the available literature.

\section{Case Report}

A 13-month-old Caucasian female from an affluent family came to our department due to progressively worsening edema of both eyelids and lower extremities over the last three weeks along with increasing fatigue and irritability. Vital signs at presentation showed temperature $36^{\circ} \mathrm{C}$, respirations $25 / \mathrm{min}$, pulses $210 / \mathrm{min}$, blood pressure $89 / 56 \mathrm{mmHg}$ and oxygen saturation $97 \%$. On physical examination, the child weighted $10.3 \mathrm{~kg}$ (up $700 \mathrm{~g}$ from her most recently recorded weight a month ago), had intense paleness without a rash, anasarca, and a hyperdynamic precordium with a $2 / 6$ systolic heart murmur.

The patient was born full-term by caesarian section and was fully immunized for age. She received iron-fortified infant formula for the first six months of her life, but over the last several months she was receiving progressively increasing amounts of fresh cow's milk that recently exceeded 1.2 liters per day. During the last three weeks, she had become progressively anorexic and was refusing to eat solid foods. On specific questioning, the parents refused consumption of non-nutritive substances from the infant, i.e., pica.

Laboratory examinations on admission showed leukocytes 15,970/ $\mu$ l (lymphocytes 69\%, neutrophils $25 \%$, monocytes $4 \%$, eosinophils $2 \%$ ), hemoglobin $3.8 \mathrm{~g} / \mathrm{dl}$, hematocrit $13.4 \%$, MCV $55.1 \mathrm{fl}, \mathrm{MCH} 15.6 \mathrm{pg}$, RDW 27\%, platelets $523,000 / \mu \mathrm{l}$, reticulocytes $0.21 \%$, glucose $90 \mathrm{mg} /$ $\mathrm{dl}$, blood urea nitrogen $14.9 \mathrm{mg} / \mathrm{dl}$, creatinine $0.2 \mathrm{mg} / \mathrm{dl}$, albumin $2 \mathrm{~g} / \mathrm{dl}$, total bilirubin 0.1 $\mathrm{mg} / \mathrm{dl}$, ferritin $1.9 \mathrm{ng} / \mathrm{ml}$ (reference range 7-140) and LDH 248 U/1. Examination of the peripheral blood smear showed microcytosis, hypochromasia, prominent anisocytosis and poikilocytosis. Several urinalyses were normal showing no proteinuria, while a stool guaiac test was weakly positive for occult blood. Serum analysis for gluten sensitivity (IgA and IgG anti tissue-transglutaminase) was negative. We did not test stool $\mathrm{a}_{1}$-antitrypsin, a frequently used marker of endogenous intestinal protein loss, because its measurement was not available to us.

After establishing intravenous access, the patient was transfused on an emergency basis with $130 \mathrm{ml}$ of compatible packed red blood cells. After the end of the transfusion, she received $20 \mathrm{~g}$ of intravenous albumin. On the second and fourth hospital days, the patient received over two hours $100 \mathrm{mg}$ of iron sucrose intravenously, treatment that was tolerated well. On physical examination, the child demonstrated substantial improvement of the anasarca already from the end of the second hospital day. She was discharged after complete resolution of the edema on the fifth hospital day weighting $9.67 \mathrm{~kg}$ (a $630 \mathrm{~g}$ decrease from the admission weight). Full blood count at discharge showed hemoglobin $8.5 \mathrm{~g} / \mathrm{dl}$, hematocrit $26.5 \%$, MCV $67.6 \mathrm{fl}$, reticulocytes $3.7 \%$, platelets $469,000 / \mu \mathrm{l}$ and albumin $3.5 \mathrm{~g} /$ dl. The family was advised to minimize milk intake to a maximum of $500 \mathrm{ml}(16.9 \mathrm{oz})$ per day and to obtain a new full blood count and serum albumin in four weeks. The patient did not receive any oral iron therapy. Repeated examinations at that time showed hemoglobin $11.2 \mathrm{~g} / \mathrm{dl}$, hematocrit $32.7 \%$, MCV $78.2 \mathrm{fl}$, reticulocytes $2.9 \%$, and serum albumin $3.6 \mathrm{~g} /$ dl. Eight months after the described events, the patient is healthy with a normal hemogram for age. A radioallergosorbent blood test at that time for allergy to cow's milk protein showed absent specific IgE.

\section{Discussion}

We present a female toddler with severe IDA associated with hypoalbuminemia and anasarca. Although the latter is a classic clinical finding of nephrotic syndrome, several urinalyses ruled out this diagnosis in our patient, who had no proteinuria.

PLE is a clinical entity associated with severe loss of serum proteins into the intestine. Normal albumin loss through the gut accounts for only around $6 \%$ of the total body albumin 
Table I. Toddlers with Severe IDA Associated with Anasarca and Hypoalbuminemia Described in the Past, and Their Hemoglobin at Clinical Presentation.

\begin{tabular}{|c|c|c|c|c|}
\hline & Author & Age & Sex & $\begin{array}{l}\text { Hemoglobin at diagnosis } \\
(\mathrm{g} / \mathrm{dl})\end{array}$ \\
\hline 1 & Sakai et $\mathrm{al}^{9}$ & 23-month-old & Female & $\mathrm{NA}^{*}$ \\
\hline 2 & Sakai et $\mathrm{al}^{9}$ & 14-month-old & Female & NA \\
\hline 3 & Hamrick $^{10}$ & 19-month-old & Female & 4.2 \\
\hline 4 & Vogelaar et al ${ }^{11}$ & 13-month-old & Female & 7 \\
\hline 5 & Anonymous $^{12}$ & 12-month-old & Female & 4.1 \\
\hline 6 & Salstrom et al ${ }^{13}$ & 16-month-old & Male & 3.1 \\
\hline 7 & Salstrom et $\mathrm{al}^{13}$ & 26-month-old & Male & 3.9 \\
\hline 8 & Lundström et al $^{14}$ & 8-month-old & Male & 5.3 \\
\hline 9 & Lundström et $\mathrm{al}^{14}$ & 9-month-old & Male & 5.7 \\
\hline 10 & Lundström et al ${ }^{14}$ & 10-month-old & Male & 6.3 \\
\hline 11 & Lundström et $\mathrm{al}^{14}$ & 10-month-old & Female & 5.3 \\
\hline 12 & Lundström et al $^{14}$ & 10-month-old & Female & 4.1 \\
\hline 13 & Lundström et $\mathrm{al}^{14}$ & 12-month-old & Female & 7.6 \\
\hline 14 & Lundström et $\mathrm{al}^{14}$ & 15-month-old & Male & 5.2 \\
\hline 15 & Lundström et $\mathrm{al}^{14}$ & 15-month-old & Female & 2.0 \\
\hline
\end{tabular}

NA*: not available (article in Japanese)

turnover, but in patients with severe PLE, it can reach up to $60 \%$ of the total albumin pool. ${ }^{6}$ Constrictive pericarditis, congestive heart failure, regional enteritis, ulcerative colitis, giant hypertrophic gastritis (Ménétrier disease), intestinal lymphangiectasia, lymphoenteric fistulas, Whipple's disease, and intestinal parasitosis are only a few examples of diseases associated with PLE. On the other hand, clinical studies and few case reports have shown excessive milk intake to be the sole cause of severe IDA associated with PLE, hypoalbuminemia and anasarca. ${ }^{7-14}$

IDA can occur irrespective of the child's socioeconomic status, and routine iron supplementation in high-risk infants and toddlers has decreased its incidence. ${ }^{1}$ Although the prevalence of IDA in industrialized countries is highest among children living below the poverty line, the child we describe was from an affluent family and the IDA was the result of chronic consumption of large amounts of unfortified commerciallyavailable pasteurized fresh cow's milk along with minimal consumption of iron-fortified solid foods due to anorexia. Worsening IDA is associated with progressive anorexia and the latter minimizes further iron intake leading to a vicious circle that culminates in life-threatening IDA. It should be noted that IDA is the $10^{\text {th }}$ most important modifiable risk factor for early death according to WHO. ${ }^{15}$

Prior published reports (summarized in Table I) have shown the median hemoglobin of patients with IDA and anasarca associated with excessive cow's milk intake to be 5.2 $\mathrm{g} / \mathrm{dl}$ (range 2-7.6) at diagnosis. As shown in the table, the patients' age at diagnosis ranged from 8 to 26 months (median age 13 months), with a slight female predominance (9 of 15 patients or $60 \%$ ).

The exact mechanism of PLE in patients with severe IDA is unknown, and remarkably enough, not all children with severe IDA due to excessive fresh cow's milk intake develop anasarca. Prior studies have shown a high incidence of abnormalities of gastrointestinal function and structure in children with nutritional IDA, such as varying degrees of chronic duodenitis and villous atrophy. ${ }^{14}$ The reversal of these abnormalities with iron treatment suggests that they represent the effects rather than the causes of IDA. Woodruff et al measured the albumin turnover in a whole-body counter in six normal infants and 
12 infants with IDA following the intravenous injection of radiolabeled albumin. Seven of the 12 patients had a rapid turnover of albumin indicative of considerable exudative enteropathy. The authors concluded that the increased albumin turnover reflects an intestinal albumin loss which does not respond to intravenous iron therapy adequate to produce a hematologic response and that withdrawal of cow's milk is necessary for resolution of the PLE. ${ }^{8}$ Lundström et al investigated 42 children aged 8-24 months with severe IDA, of whom eight also had hypoproteinemia and edema, and 13 age-matched controls. Children with IDA had excessive fecal loss of radiolabed iron, albumin, or both. They were all treated with an oral ferrous iron preparation and the edema, hypoproteinemia, and IDA resolved despite lack of evidence from intestinal biopsy specimens that immunologically mediated hypersensitivity was implicated. ${ }^{14}$ In vitro blood testing in our patient was also not suggestive of IgE-mediated cow's milk protein allergy.

We elected to use off label intravenous iron sucrose (in the US approved for children aged $\geq 2$ years) after an initial transfusion of packed erythrocytes. Although oral iron is effective in correcting the IDA associated with excessive consumption of cow's milk, oral iron therapy requires good patient compliance for several months, it is frequently poorly tolerated because of its metallic aftertaste and, in situations associated with gastrointestinal bleeding, iron loss may be greater than the oral iron supply. Instead, intravenous iron has perfect bioavailability, by bypassing the hepcidin-ferroportin pathway that controls iron absorption and corrects the IDA faster than any oral iron therapy. ${ }^{16}$ The patient's severe IDA was safely and fully corrected within four weeks after intravenous iron administration, although the anasarca corrected much faster. It should be noted that the development of generalized edema is related to the iron deficiency itself, and not to the anemia, since severely anemic but not iron deficient patients do not develop anasarca. Thus, it is anticipated that the correction of the iron deficit will lead to resolution of the anasarca earlier than the correction of anemia, which was exactly the case in our patient.

In conclusion, excessive consumption of fresh cow's milk is associated with iron deficiency and IDA. In severe cases, anasarca can develop and be the main reason for seeking medical attention. Pediatricians should be aware of the association of severe IDA and anasarca, and should screen infants in their practice for IDA at the age of 12 months or sooner, if risk factors, such as consumption of fresh cow's milk before their first birthday, are present. Finally, front-line use of intravenous iron should strongly be considered in infants and children with severe IDA, since this underutilized form of iron therapy appears to be safe and extremely effective.

\section{References}

1. Baker RD, Greer FR; Committee on Nutrition American Academy of Pediatrics. Diagnosis and prevention of iron deficiency and iron-deficiency anemia in infants and young children (0-3 years of age). Pediatrics 2010; 126: $1040-1050$.

2. Powers JM, Buchanan GR. Diagnosis and management of iron deficiency anemia. Hematol Oncol Clin North Am 2014; 28: 729-745.

3. Looker AC, Dallman PR, Carroll MD, Gunter EW, Johnson CL. Prevalence of iron deficiency in the United States. JAMA 1997; 277: 973-976.

4. Gupta PM, Perrine CG, Mei Z, Scanlon KS. Iron, anemia, and iron deficiency anemia among young children in the United States. Nutrients 2016; 8. pii: E330.

5. Worldwide prevalence of anaemia 1993-2005: WHO global database on anaemia. de Benoist B, McLean E, Egli I, Cogswell M (eds). World Health Organization, Geneva: 2008: 7-13.

6. Waldmann TA, Wochner RD, Strober W. The role of the gastrointestinal tract in plasma protein metabolism. Studies with 51Cr-albumin. Am J Med 1969; 46 : 275-285.

7. Woodruff CW, Clark JL. The role of fresh cow's milk in iron deficiency. I. Albumin turnover in infants with iron deficiency anemia. Am J Dis Child 1972; 124: 18-23.

8. Woodruff CW, Wright SW, Wright RP. The role of fresh cow's milk in iron deficiency. II. Comparison of fresh cow's milk with a prepared formula. Am J Dis Child 1972; 124: 26-30.

9. Sakai Y, Sakai R, Yoshida N, Miyazaki S. Iron deficiency anemia and hypoproteinemia induced by fresh cow's milk. Rinsho Ketsueki 1992; 33: 1691-1696.

10. Hamrick HJ. Whole cow's milk, iron deficiency anemia, and hypoproteinemia: an old problem revisited. Arch Pediatr Adolesc Med 1994; 148: 1351-1352.

11. Vogelaar JL, Loar RW, Bram RJ, Fischer PR, Kaushik R. Anasarca, hypoalbuminemia, and anemia: what is the correlation? Clin Pediatr (Phila) 2014; 53: 710-712.

12. Anonymous. Iron-deficiency anemia with hypoproteinemia in an infant. Nutr Rev. 1989; 47: 48-50. 
13. Salstrom JL, Kent M, Liang X, Wang M. Toddlers with anasarca and severe anemia: a lesson in preventive medicine. Curr Opin Pediatr 2012; 24: 129-133.

14. Lundström U, Perkkiö M, Savilahti E, Siimes M. Iron deficiency anaemia with hypoproteinaemia. Arch Dis Child 1983; 58: 438-441.
15. Ezzati M, Lopez AD, Rodgers A, Vander Hoorn S, Murray CJ. Comparative Risk Assessment Collaborating Group. Selected major risk factors and global and regional burden of disease. Lancet. 2002; 360: 13471360 .

16. Mantadakis E. Advances in pediatric intravenous iron therapy. Pediatr Blood Cancer 2016; 63: 11-16. 